\title{
Diseño de un instrumento para el análisis de las competencias del logopeda escolar y del maestro de Audición y Lenguaje
}

\author{
María Salvadora Cifuentes Lardín ${ }^{1}$ y Juan Pedro Martínez Ramón ${ }^{2}$ \\ ${ }^{1}$ Graduada en Logopedia; ${ }^{2}$ Universidad de Murcia (España)
}

\begin{abstract}
El presente estudio se llevó a cabo por las incongruencias encontradas sobre las competencias del logopeda y del maestro especialista en Audición y Lenguaje (AL) y la dificultad para valorar dicho fenómeno, surgiendo la necesidad de delimitar las diferencias entre ambos, así como saber qué entendían los profesionales de la educación sobre esta cuestión. Por ello, el objetivo de la presente investigación fue el diseño y puesta en marcha de un cuestionario para analizar los perfiles profesionales del logopeda y del AL. El cuestionario fue suministrado a un total de 104 personas pertenecientes a ocho colegios públicos y concertados, un centro de atención temprana, una asociación y un centro privado; todos ellos desarrollaban su labor profesional en el municipio de Totana (Murcia, España). Dicho instrumento tuvo un total de 29 ítems cerrados llevándose a cabo la validación del mismo. Tras la administración y recogida de datos, se valoraron los resultados mediante el programa estadístico SPSS, a través del cual se realizó una evaluación de la correlación ítem-total, el coeficiente de fiabilidad y la validación empírica mediante las correlaciones. De acuerdo con los resultados obtenidos, el grupo analizado carece de nociones sobre las diferencias de estas titulaciones y las consideran como profesiones similares.
\end{abstract}

Palabras clave: Logopedia, Audición y Lenguaje, perfil profesional, cuestionario.

Design of an instrument to analysis the competences of the speech therapist and the specialist teacher in hearing and language. The present study was carried out by the inconsistences found on the competences of the speech therapist and the specialist teacher in hearing and language (AL) and the difficulty to assess this phenomenon, arising the need to delimit the diferences between both, as well as to know what they understood education professionals on this issue. Therefore, the aim of this investigation was the design and implementation of a questionnaire to analyze the profiles of the speech therapist and the AL. The questionnaire was provided to a total of 104 people belonging to eight public and subsidized schools, an early care center, an association and a private center; all of them developed their professional work in the municipality of Totana (Murcia, Spain). This instrument had a total of 29 closed items being carried our validation. After the administration and data collection, the results were evaluated through the statistical program SPSS, through which an evaluation of the item-total correlation, the reliability coefficient and the empirical validation through the correlations were performed. In agreement with results, the analyzed group lacks notions about the differences of these degrees and consider them as similar professions.

Keywords: Speech therapy, hearing and language, professional profile, questionnaire.

Correspondencia: María Salvadora Cifuentes Lardín. Res. Espuña. C/ Ágata $\mathrm{n}^{\mathbf{0}}$ 32, bz-31. C.P.: 30850. Totana, Murcia (España).E-mail: marisa_336@ hotmail.com 
La Logopedia puede ser definida como la ciencia encargada de prevenir, evaluar, diagnosticar y realizar una intervención sobre los trastornos del lenguaje, voz, habla, comunicación, audición y deglución. Esto incluye atender a una población de gran envergadura, proveniente de sectores tan dispares como el sanitario, social y el educativo, sin olvidar el ámbito de la investigación, inherente a todos ellos (Martín, 2010; Peña-Casanova, 2001). En España, la descripción reglada de las competencias que conlleva la labor logopédica pueden ubicarse en 1985, cuando la Universidad de Madrid comenzó los cursos de formación de Audición y Lenguaje a un nivel oficial universitario, en los que estaban inmersos profesionales de nueva diversa índole. Estos cursos se fueron extendiendo a otras universidades y en 1991 se implantó la Diplomatura en España.

A consecuencia de esta corta trayectoria que tiene la Logopedia, ésta se enfrenta a numerosos retos, algunos de ellos de carácter administrativo si bien es cierto que en la actualidad existen logopedas en centros educativos privador/concertados formando parte del personal no docente. Según Peñafiel (2010), las funciones generales del logopeda respecto al centro giran en torno a la participación en la elaboración del Proyecto Educativo del Centro, colaboración con el tutor y el profesor de apoyo en la programación del aula, elaboración conjunta con el tutor y el de apoyo de las adaptaciones curriculares individualizadas precisas, facilitación del asesoramiento y orientación pertinentes en relación a las adaptaciones metodológicas y organizativas del aula, evaluación en coordinación con el tutor y el resto de la comunidad educativa, colaboración con los tutores para potenciar en el aula los aspectos comunicativo-lingüísticos, entre otras. Pero dependiendo del tipo de escuela, y del servicio para el que está contratado, sus funciones específicas variarían desde tareas de prevención, diagnóstico o evaluación, tratamiento, asesoramiento, seguimiento de casos, charlas, así como coordinación con asociaciones de madres y padres de alumnos, principalmente.

Por otro lado, los maestros especialistas en Audición y Lenguaje (AL), comenzaron a incorporarse a los centros educativos también en 1985 a través del Real Decreto de 6 de marzo en el que se marcaban las pautas de ordenación de la Educación Especial y es a partir de ese momento cuando comienzan a integrar en los centros a alumnos con minusvalías (psíquicas, sensoriales y motrices), pero no es hasta la publicación de la LOGSE cuando este profesional comienza a ser más habitual en las aulas, encargándose de la estimulación de la adquisición del lenguaje oral de los niños.

Según el Real Decreto 1440/1991 del 30 de agosto, el AL se dedica a promover y desarrollar la prevención de los problemas del lenguaje, a potenciar las capacidades comunicativo-lingüísticas y a la solución de problemas específicos de lenguaje y comunicación en un centro educativo. Por lo que sus funciones van desde atender a todos los alumnos que presentan dificultades tanto de lenguaje como de comunicación, realizar adaptaciones curriculares o servir de apoyo y orientación al resto de profesionales del centro (Gallardo y Gallardo, 1993). 
Se observa una corriente de docentes que consideran que la labor de los AL se debe desarrollar fuera del horario lectivo puesto que consideran que sus tareas son externas al colegio. En este sentido, la Ley Orgánica 2/2006 junto con el Instituto Superior de Formación y Recursos en red para el profesorado, estableció que sus funciones era fomentar la prevención, desarrollar las capacidades comunicativo-lingüísticas y solucionar los problemas de dicho ámbito, excluyendo de esta manera los trastornos de la voz, la audición, el habla y la deglución, considerando estas patologías tratables exclusivamente por los logopedas. En cambio, Peñafiel (2010), estableció como funciones propias del AL aspectos más concretos del ámbito escolar como la gestión de informes provenientes de los Equipos de Orientación Educativa y de gabinetes, coordinarse con Atención temprana, impulsar los programas de prevención, realizar tratamiento y asesorar a la comunidad educativa.

Por todo ello, una de las cuestiones que se recoge en esta investigación es saber si los AL se sienten realmente satisfechos con su trabajo. La insatisfacción por parte de los docentes viene determinada sobre todo por la formación recibida, situación que han ido solventado de forma autónoma mediante cursos de formación, siendo necesario definir las funciones (Luque y Carrión, 2013a, 2013b, 2013c). Otras de las trabas con las que se tienen que enfrentar estos maestros vienen determinadas por las características inherentes de sus funciones, centradas en el proceso de enseñanza-aprendizaje.

En el año 2009 la Organización de Cooperación y el Desarrollo Económico (OCDE) publicó que había un porcentaje relevante de profesores que consideran no estar formados para la situación actual del ámbito escolar (Pedró, 2009). Algo más alejado en el tiempo, desde mediados de los años noventa, se ha promovido una escuela inclusiva en la que se suministrara una respuesta educativa eficaz, surgiendo la necesidad de involucrar la logopedia al contexto escolar para dar esa respuesta a las alteraciones del lenguaje que pueden surgir en esta edad. El profesional que realiza esta inclusión es el AL, no pudiendo tratar aquellas dificultades que por su complejidad son incompatibles en el currículum educativo, como pueden ser las disfonías o la deglución atípica. Sí que es cierto que dentro de los trastornos más frecuentes que se dan en las escuelas, se encuentran las hipoacusias o la disfemia, al considerarse dos trastornos muy complejos por sus connotaciones fisiológicas/anatómicas es importante incluir la logopedia en la escuela ya que es el único profesional especializado en los trastornos de habla y audición (Peñafiel y Fernández, 2010; Sos y Sos, 2011).

En el artículo de Rosell (1996), se hizo una comparativa con respecto al currículo oficial de estas dos titulaciones que son muy semejantes y que a la vez están muy enfrentadas. A pesar de que hay ciertas semejanzas, también se observan diferencias, aunque estas desigualdades son más o menos cercanas siendo cuantitativas o cualitativas. A nivel cuantitativo, se observa que en el plan de estudio del especialista de AL, éste tiene que realizar ciertas materias comunes a todos los títulos de magisterio haciendo que se 
limiten los créditos de la especialidad. A nivel cualitativo, en lo que a la intervención se refiere, las asignaturas obligatorias del título de logopedia vienen determinadas por "trastornos del habla, desarrollo, y voz", mientras que en la especialidad de AL aparecen como "tratamientos educativos" de estos mismos trastornos, esto hace que los AL desarrollen capacidades para llevar a cabo un tratamiento en el ámbito educativo, sin poder llegar a extenderlo a otro contexto y por consiguiente no sería una rehabilitación total. Otro aspecto importante a destacar en estas diferencias del currículo, son las que están relacionadas con la evaluación. Al igual que ocurre con el tratamiento, la evaluación que pude llevar AL es educativa relacionada con los estándares del currículo educativo, en cambio, el logopeda desarrolla una evaluación y diagnóstico del trastorno. No obstante, al margen de las diferencias, es necesario prestar atención también a aquellos puntos que unen el trabajo de AL y logopeda, en aras de dar una respuesta integral al alumnado (Castegón y España, 2004).

Dicho esto, la presente investigación tiene como objetivo diseñar y poner en marcha un cuestionario para analizar los perfiles profesionales del logopeda y AL. Dicho cuestionario pretende ser un instrumento para indagar información sobre la labor profesional de ambos colectivos.

\section{MÉTODO}

\section{Participantes}

Los participantes que formaron parte de este estudio fueron un total de 104 personas, pertenecientes a 8 centros del municipio de Totana (Murcia), de los cuales $18.3 \%$ eran hombres y $81.7 \%$ eran mujeres. La franja de edad de la mayoría de los participantes, se encontraba comprendida entre los intervalos de 26 a 35 y 35 y 46 , ya que la ponderación de los valores asignados correspondientes a esta edad es de 2 y 3 respectivamente. Por consiguiente la media de edad es de 2.86 y cuya desviación típica es .875. Como criterios de inclusión y exclusión, se tomó en cuenta a docentes fijos e interinos de las etapas de Educación Infantil y Primaria. Por otro lado, se excluyó a uno de los centros públicos del municipio porque el Equipo Directivo así lo decidió. A todos ellos, se les comentó que sería de los datos más relevantes del estudio una vez finalizado.

\section{Instrumento}

Se elaboró una encuesta de preguntas cerradas que comparaba los perfiles profesionales de los logopedas y los AL. Este instrumento pretendía conocer datos sobre el trabajo que desarrollan ambos perfiles, conocimiento que se tiene sobre la existencia de ambas titulaciones, así como investigar sobre los diferentes trastornos sobre los que pueden actuar. 
La encuesta "Logopedia v/s AL" pretendía medir el impacto que tiene la Logopedia sobre el mundo educativo. Contenía un total de 29 ítems y cuyas respuestas están agrupadas según el grado de satisfacción ( 0 totalmente en desacuerdo; 1 en desacuerdo; 2 de acuerdo; 3 totalmente de acuerdo). Las variables que se emplearon son de tipo cuantitativo puesto que se distinguen unos valores ordenados siguiendo la escala establecida. A lo largo de la secuencias de estos ítems, se llevó una comparativa directa sobre las funciones de los logopedas y de los AL así como valorar el conocimiento que se tenía sobre estos profesionales.

\section{Procedimiento}

Estudio descriptivo de diseño ex post facto. La validación del instrumento se llevó a cabo mediante la colaboración de seis docentes. Las aportaciones que realizaron fueron de tipo constructivo con referencia todas ellas a la agrupación de los ítems o la forma de redacción de los mismos.

Todos los participantes pertenecen al ámbito escolar, puesto que trabajan en centros públicos o concertados donde la mayoría de la población que es atendida es infantil. Se veló en todo momento por garantizar la voluntariedad, anonimato y confidencialidad de los datos de todos los colaboradores.

\section{Análisis estadístico}

Para el análisis del estudio se utilizó el paquete estadístico Statistical Package for the Social Sciences 19 (SPSS). En el cual se llevó a cabo una evaluación de los ítems, la fiabilidad y la validez. La interpretación de los ítems se llevó a cabo mediante el análisis de homogeneidad. Para realizar este estudio, se observó la media, la desviación típica, la correlación de elementos corregidos y Alfa de Cronbach.

Con respecto al análisis de fiabilidad, se evalúa el Alfa de Cronbach y el Índice de Dos Mitades de Guttman. Ambos índices varían entre 0-1, cuanto más se aproximen al 1 mayor fiabilidad, en cambio si el resultado fuese inferior a 7, descartaríamos este Índice, ya que lo consideraríamos un test poco fiable para valorar los perfiles profesionales de los logopedas y de los AL. En los resultados de la validez se empleó la Correlación de Pearson. Estos son los criterios que usaremos en el apartado de resultados para llevar a cabo la evaluación del test. 


\section{RESULTADOS}

\section{Interpretación del análisis de ítems}

Partiendo del índice de dificultad, no se descarta ningún ítem. En la tabla 1, se ve que todos los valores se encuentran entre los intervalos de .30 y .70 . Con respecto a la media de las puntuaciones recogidas, en esta misma, se observa que varía desde el 1.03 al 2.97, por lo que la desviación típica es alta, así que los participantes de este experimento consideran que si hay diferencias en estos dos perfiles.

Tabla 1. Correlación ítem-total: Perfil del Logopeda

\begin{tabular}{|c|c|c|c|}
\hline & Media & $\begin{array}{l}\text { Correlación } \\
\text { elemento-total } \\
\text { corregido }\end{array}$ & $\begin{array}{l}\text { Alfa de Cronbach si se } \\
\text { elimina el elemento }\end{array}$ \\
\hline \multicolumn{4}{|l|}{ Perfil del Logopeda } \\
\hline 1. Conozco el perfil profesional & 2.03 & .311 & .837 \\
\hline $\begin{array}{l}\text { 2. Conozco las competencias que deben } \\
\text { trabajar }\end{array}$ & 1.82 & .387 & .835 \\
\hline $\begin{array}{l}\text { 3. Deben estos profesionales estar al día de } \\
\text { todas las novedades en investigación. }\end{array}$ & 2.87 & .232 & .839 \\
\hline 4. Es importante la formación continua. & 2.97 & .305 & .840 \\
\hline $\begin{array}{l}\text { 5. Hay que tener en cuenta la Práctica Basada } \\
\text { en la Evidencia para planificar la intervención. }\end{array}$ & 2.67 & .362 & .836 \\
\hline $\begin{array}{l}\text { 6. Trabajan las mismas patologías (voz, } \\
\text { audición, lenguaje, deglución, habla y } \\
\text { comunicación). }\end{array}$ & 2.11 & .515 & .830 \\
\hline 7. La formación académica es similar & 1.79 & .359 & .836 \\
\hline
\end{tabular}

Nota. $M$ (Media); DT (Desviación Típica); Elemento-Total (Correlación elemento-total corregido); Alpha Cronbach (Alfa de Cronbach si se elimina el elemento).

Tabla 2. Correlación ítem-total: Perfil del Maestro AL

\begin{tabular}{lcccc}
\hline \multicolumn{1}{c}{ Perfil del Maestro AL } & $M$ & $D T$ & $\begin{array}{c}\text { Elemento- } \\
\text { Total }\end{array}$ & Alpha Cronbach \\
\hline 1. Conozco el perfil profesional & 2.36 & .708 & .568 & .829 \\
\hline $\begin{array}{l}\text { 2. Conozco las competencias que deben } \\
\text { trabajar }\end{array}$ & 2.21 & .755 & .511 & .831 \\
\hline $\begin{array}{l}\text { 3. Deben estos profesionales estar al día } \\
\text { de todas las novedades en investigación. }\end{array}$ & 2.77 & .462 & .354 & .837 \\
\hline 4. Es importante la formación continua. & 2.93 & .250 & .220 & .840 \\
\hline $\begin{array}{l}\text { 5. Hay que tener en cuenta la Práctica } \\
\text { Basada en la Evidencia para planificar la } \\
\text { intervención. }\end{array}$ & 2.61 & .613 & .372 & .836 \\
\hline $\begin{array}{l}\text { 6. Trabajan las mismas patologías (voz, } \\
\text { audición, lenguaje, deglución, habla y } \\
\text { comunicación). }\end{array}$ & 2.07 & .793 & .421 & .834 \\
\hline 7. La formación académica es similar & 1.84 & .711 & .411 & .834 \\
\hline
\end{tabular}

Nota. $M$ (Media); DT (Desviación Típica); Elemento-Total (Correlación elemento-total corregido); Alpha Cronbach (Alfa de Cronbach si se elimina el elemento) 
CIFUENTES y MARTÍNEZ. Competencias del logopeda escolar y del maestro AL

Tabla 3. Correlación ítem-total. Ambos perfiles

\begin{tabular}{lllll}
\hline Ambos perfiles & M & DT & $\begin{array}{c}\text { Elemento- } \\
\text { Total }\end{array}$ & $\begin{array}{c}\text { Alpha } \\
\text { Cronbach }\end{array}$ \\
\hline $\begin{array}{l}\text { 8. Las funciones que desarrollan los AL, cumplen } \\
\text { con las expectativas pensadas. }\end{array}$ & 2.44 & .620 & .175 & .841 \\
\hline $\begin{array}{l}\text { 9. La formación académica que reciben los AL, } \\
\text { permite hacer frente a las dificultades o trastornos de } \\
\text { los alumnos con los que se enfrenta. }\end{array}$ & 2.10 & .676 & .319 & .837 \\
\hline $\begin{array}{l}\text { 10. Hay intrusismo por parte de los logopedas en el } \\
\text { ámbito educativo. }\end{array}$ & 1.33 & .870 & .264 & .840 \\
\hline $\begin{array}{l}\text { 11. Hay recursos suficientes en los centros } \\
\text { educativos para intervenir el AL }\end{array}$ & 1.30 & .782 & .400 & .834 \\
\hline $\begin{array}{l}\text { 12. El AL está preparado para trabajar en un } \\
\text { gabinete logopédico. }\end{array}$ & 1.97 & .706 & .548 & .830 \\
\hline $\begin{array}{l}\text { 13. Los logopedas son importantes en el centro } \\
\text { escolar. }\end{array}$ & 2.30 & .901 & .478 & .831 \\
\hline $\begin{array}{l}\text { 14. El logopeda debe formar parte de la comunidad } \\
\text { educativa. }\end{array}$ & 2.39 & .781 & .420 & .834 \\
\hline $\begin{array}{l}\text { 15. El logopeda mejora la calidad de la enseñanza. } \\
\text { 16. El logopeda está preparado para trabajar en un } \\
\text { centro educativo. }\end{array}$ & 2.36 & .775 & .482 & .831 \\
\hline $\begin{array}{l}\text { 17. Los programas de intervención logopédica son } \\
\text { importantes para intervenir en la escuela. }\end{array}$ & 2.33 & .724 & .358 & .836 \\
\hline $\begin{array}{l}\text { 18. Hay intrusismo por parte de los AL en } \\
\text { desarrollar tareas concretas en aspectos del } \\
\text { desarrollo de la comunicación, habla o lenguaje. }\end{array}$ & 1.43 & .865 & .332 & .837 \\
\hline $\begin{array}{l}\text { 19. El logopeda cuenta con recursos suficientes para } \\
\text { intervenir en distintas patologías. }\end{array}$ & 1.90 & .676 & .170 & .842 \\
\hline $\begin{array}{l}\text { 20. Los AL y los logopedas son lo mismo. } \\
\text { 21. Los AL y los logopedas son dos perfiles que se } \\
\text { complementan. }\end{array}$ & 2.46 & .621 & .111 & .843 \\
\hline $\begin{array}{l}\text { 22. Las Universidades deben unificar esas dos } \\
\text { titulaciones. }\end{array}$ & 1.98 & .975 & .275 & .840 \\
\hline $\begin{array}{l}\text { Nota. } M \text { (Media); DT (Desviación Típica); Elemento-Total (Correlación elemento-total corregido); Alpha } \\
\text { Cronbach (Alfa de Cronbach si se elimina el elemento) }\end{array}$ & & .894 & .444 & \\
\hline
\end{tabular}

\section{Interpretación del coeficiente de fiabilidad}

Los estadísticos de fiabilidad en los 29 ítems de la encuesta presentan en el Alfa de Cronbach una puntuación general de .841, y el resultado de Dos Mitades de Guttman es de .511 siendo excluidos a 43 personas del grupo de 104, quedando con un total de 61 casos válidas.

\section{Interpretación de la validación empírica}

En cuanto a los coeficientes de correlación, podemos interpretar a simple vista que las variables en su mayoría se encuentran altamente relacionadas. Véase tabla 2.

Comparando las variables "edad", "AL", "Logopeda" y "Ambos perfiles", podemos observar claramente que el mayor índice de correlación se concentra en la variable "Ambos perfiles" siendo su índice de correlación de .874, seguido de "Logopeda" .718 . 
CIFUENTES y MARTÍNEZ. Competencias del logopeda escolar y del maestro AL

Tabla 4. Correlaciones

\begin{tabular}{|c|c|c|c|c|c|c|}
\hline & & Edad & $\begin{array}{c}\text { Ítems perfil } \\
\mathrm{AL}\end{array}$ & $\begin{array}{l}\text { Ítems perfil } \\
\text { Logopedas }\end{array}$ & $\begin{array}{l}\text { Ítems ambos } \\
\text { perfiles }\end{array}$ & Total \\
\hline \multirow{3}{*}{ Edad } & $\begin{array}{l}\text { Correlación de } \\
\text { Pearson }\end{array}$ & 1 & .189 & .004 & -.051 & .063 \\
\hline & Sig. (Bilateral) & & .084 & .969 & 669 & .630 \\
\hline & $N$ & 104 & 84 & 88 & 72 & 61 \\
\hline \multirow{3}{*}{$\begin{array}{l}\text { Ítems para el } \\
\text { perfil AL }\end{array}$} & $\begin{array}{l}\text { Correlación de } \\
\text { Pearson }\end{array}$ & .189 & 1 & $.626^{* *}$ & $.358^{* *}$ & $.713^{* *}$ \\
\hline & Sig. (Bilateral) & .084 & & .000 & .004 & .000 \\
\hline & $N$ & 84 & 84 & 81 & 64 & 61 \\
\hline \multirow{3}{*}{$\begin{array}{l}\text { Ítems para el } \\
\text { perfil Logopeda }\end{array}$} & $\begin{array}{l}\text { Correlación de } \\
\text { Pearson }\end{array}$ & .004 & $.626^{* *}$ & 1 & $.406^{* *}$ & $.718^{* *}$ \\
\hline & Sig. (Bilateral) & .969 & .000 & & .001 & .000 \\
\hline & $\bar{N}$ & 88 & 81 & 88 & 65 & 61 \\
\hline \multirow{3}{*}{$\begin{array}{l}\text { Ítems para ambos } \\
\text { perfiles }\end{array}$} & $\begin{array}{l}\text { Correlación de } \\
\text { sPearson }\end{array}$ & -.051 & $.358^{* *}$ & $.406^{* *}$ & 1 & $.874^{* *}$ \\
\hline & Sig. (Bilateral) & .669 & .004 & .001 & & .000 \\
\hline & $\bar{N}$ & 72 & 64 & 65 & 72 & 61 \\
\hline \multirow{3}{*}{ Total de ítems } & $\begin{array}{l}\text { Correlación de } \\
\text { Pearson }\end{array}$ & .063 & $.713^{* *}$ & $.718 * *$ & $.874^{* *}$ & 1 \\
\hline & Sig. (Bilateral) & .630 & .000 & .000 & .000 & \\
\hline & $N$ & 61 & 61 & 61 & 61 & 61 \\
\hline
\end{tabular}

Todo ello nos representa el hecho de que ambos perfiles se encuentran en una correlación positiva alta. Por lo tanto, si el perfil de logopeda se viera modificado en su sentido, el del AL lo haría en la misma dirección. Esto lleva a pensar que están estrechamente vinculados o ligados el perfil del logopeda con el perfil de AL, sin darse una clara distinción entre ellos a día de hoy según las muestras tomadas en este estudio.

\section{DISCUSIÓN Y CONCLUSIONES}

La finalidad de este estudio ha sido llevar a cabo una investigación empírica sobre el conocimiento que tienen docentes y logopedas sobre la labor que desempeñan los AL y los logopedas en el ámbito educativo mediante el análisis de estos perfiles. En relación a los ítems que se aprecian en la tabla 1 , se observa que ninguno de los enunciados supera el valor de .7, esto se debe a que no existe una gran correlación entre ellos debido a que los participantes no entendía la idea fundamental de esta encuesta que era el de analizar las diferencias de dos profesionales, considerando a estas titulaciones como sinónimas y que dependiendo del contexto, se utilizaba una nomenclatura u otra. Dentro de la media que se obtiene con respecto a análisis de ítems comprobamos que su resultado es alto. Por lo que asume que los participantes en esta encuesta tienen una opinión difusa con respecto a los enunciados que se le planteaban, o no llegaban a comprender las diferencias que se proponían entre ambos perfiles. También se observa como en la encuesta los marcadores del Alfa de Cronbach tienen una puntuación general de .841 y en 
cambio, el de Dos Mitades de Guttman, presenta un valor inferior a .8, concretamente, .511. Por lo tanto, se continúa utilizando esta escala ya que presenta buena fiabilidad aunque se podría llegar a mejorar.

Con relación al análisis de la validación, al tratarse de una encuesta con 29 ítems distribuidos en tres campos o bloques, se configuraron tres subescalas (Perfil del Maestro de Audición y Lenguaje, Perfil del Logopeda, Ambos perfiles), se llevó a cabo una interpretación individual de cada una de ellas y otra general. De acuerdo con los resultados obtenidos en la validez, no se reflejan diferencias significativas en ninguna subescala, así como tampoco en el resultado total de la validez. Estos resultados pueden ser debidos a la poca información que se tiene sobre los matices de los ítems, considerando que ambas preguntas hacía referencia a la misma persona. Por lo que, se sobreentiende que los conocimientos previos de todos los participantes sobre este tema eran escasos.

Si bien se obtienen los objetivos marcados, es necesario seguir profundizando en las funciones de uno y otro colectivo para clarificarlas tratando de definir lo que es propio de uno y otro (Gallardo y Gallardo, 1993; Tejero, 2011; González, 2013; Peñafiel y Fernández, 2010). En este contexto, la colaboración entre el maestro y el logopeda resulta fundamental para asegurar una respuesta multidimensional (Castejón y España, 2004). Las funciones y la labor de cada profesional son percibidas de forma errónea por la comunidad educativa. Además, tanto el análisis previo como el empírico reflejan que el logopeda no se encuentra inmerso dentro del sistema educativo si bien realiza algunas funciones a través del mismo mediante convenios.

Con respecto a las limitaciones y las futuras líneas de investigación, al no existir una encuesta estandarizada de esta temática, se procedió a su elaboración y posterior validación. Este hecho supuso dejar aspectos sin tener en cuenta y que posteriormente en al análisis factorial se vio que era relevantes para su análisis. Otro aspecto fue la ausencia de información teórica de este tema. Por ello, quizás la relevancia de este estudio radica en el hecho de que, al existir escasas referencias bibliográficas previas y datos empíricos sobre esta temática, esta investigación puede ser utilizada como referente de otras posteriores con relación a los AL y la logopedia escolar, considerándose la posibilidad de aumentar el número de participantes y la zona geográfica de cara a aumentar el potencial de generalización de los resultados y suscitar estudios comparativos. Por ello, es posible afirmar que este trabajo supone una primera aproximación a una visión global de esta problemática educativa sirviendo de base para futuras líneas de investigación. Las conclusiones suponen una reflexión sobre la necesidad de formar, informar y publicitar a la comunidad educativa sobre las competencias y funciones de cada uno. 


\section{REFERENCIAS}

Castejón, L.A., y España, Y. (2004). La colaboración logopeda-maestro: hacia un modelo inclusivo de intervención en las dificultades del lenguaje. Revistas de Logopedia, Foniatría y Audiología, 24(2), 55-56.

Competencias profesionales docentes y detección de necesidades de formación (s.f). Recuperado de: http://servicios.educarm.es/cnice/Comprof/WebDEF_02/puestos.pdf

Decreto nº198/2014, de 5 de septiembre, por el que se establece el currículo de la Educación Primaria en la Comunidad Autónoma de la Región de Murcia. Boletín oficial de la Región de Murcia, 6 de septiembre de 2014 núm. 206, pp. 33054-33556. Recuperado de: https://www.borm.es/borm/documentos?obj=anu\&id=713895

Documento del perfil profesional del logopeda (s.f). Recuperado de: http//www.coloan.org/downloads/perfil_profesional_logopeda.pdf

Fernández Batanero, J.M. y Rodríguez-Martín, A. (2017). TIC y diversidad funcional: conocimiento del profesorado. European Journal of Investigation in Health, Psychology and Education, 7(3), $157-165$.

Gallardo, J.R., y Gallardo J.L. (1993). Manual de Logopedia escolar. Un enfoque práctico. Málaga: Aljibe.

González, E (2013). El maestro en audición y lenguaje como recurso personal integrado en el centro. Publicaciones Didácticas, 34, 52-58.

González-Gil, F., Martín-Pastor, E., Flores Robaina, N., Jenaro Río, C., Poy Castro, R., y Gómez-Vela, M. (2013). Inclusión y convivencia escolar: análisis de la formación del profesorado. European Journal of Investigation in Health, Psychology and Education 3(2), $125-135$.

Luque, A., y Carrión, J.J. (2011) The teacher of hearing and lenguaje: an analysis of their itinerant model. European Journal of Investigation in Health, Psychology and Education 1(3), 95-103.

Luque, A., y Carrión, J. (2013a). Metodología y Recursos del Maestro Itinerante de Audición y Lenguaje. Electronic Journal of Research in Educational Psychology, 11(2), 201-526.

Luque, A., y Carrión, J. (2013b). El maestro de audición y lenguaje: formación, experiencia previa y acceso. Revista Electrónica Interuniversidades de Formación del Profesorado, 16(2), 39-54.

Luque, A., y Carrión, J.J. (2013c). Análisis del grado de satisfacción profesional del maestro de audición y lenguaje itinerante. European Journal of Education and Psychology, 6(1), 55-68.

Luque, A., y Carrión, J.J. (2014). Análisis de la actuación docente del maestro de audición y lenguaje en Almería. Revista de Logopedia, Foniatría y Audiología, 34, 68-80.

Martín, B. (2010). El logopeda en la escuela: ¿dentro o fuera del aula? Boletín Aelfa, 10(2), 35-36.

Peaña-Casanova, J. (2001). Manual de Logopedia (pp. 461-476). Barcelona: Elsevier Masson.

Peñafiel, F., y Fernández, J.D. (Coords.). (2010). Cómo intervenir en logopedia escolar. Resolución de casos prácticos (pp. 167-228). Madrid: Editorial CCS.

Rosell, V. (1996). Perfil del logopeda. Revistas de logopedia, foniatría y audiología, 16(4), 209-216.

Sos, A., y Sos, M.L. (2011). Logopedia práctica (pp. 7-24). Madrid: Wolters Kluwer.

Tejero, J.M. (2010). Las funciones de los logopedas en los centros de desarrollo infantil y atención temprana. Boletín Aelfa, 10(2), 37-38.

Recibido: 21 de noviembre de 2017

Recepción Modificaciones: 16 de enero de 2018

Aceptado: 23 de febrero de 2018 